Martin Henry, 'Le péché originel : un héritage défectueux', Etudes Théologiques et Religieuses, Tome 83 (2008/1), 15-25

\title{
LE PÉCHÉ ORIGINEL : UN HÉRITAGE DÉFECTUEUX
}

La doctrine du péché originel a marqué d'une manière négative non seulement la théologie, mais, s'il faut en croire Nietzsche, la civilisation occidentale tout entière. C'est en général à saint Augustin qu'est reprochée la sombre tradition qui entoure l'idée du péché originel, notamment, de par le lien qu'il établit entre la procréation et la transmission du péché originel. Martin HENRY ${ }^{*}$ suggère ici que malgré son pessimisme apparent, la doctrine doctrine du péché originel pourrait bien recéler un message inattendu d'espérance ${ }^{1}$.

D’après certains des commentateurs les plus autorisés de ces deux derniers siècles, la doctrine du péché originel (avec la promesse du rachat qu’elle entraîne) serait non seulement un des enseignements essentiels du christianisme, mais le noyau incontournable de celui-ci, qui sans cela perdrait sa cohérence et son intégrité. Pour le Schopenhauer du Monde comme volonté et représentation, par exemple, c'est une evidence : «En définitive, la doctrine du péché originel (affirmation de la volonté) et de la rédemption (négation de la volonté) est la vérité capitale qui forme, pour ainsi dire, le noyau du christianisme ; tout le reste n'est le plus souvent que figure, enveloppe ou hors d'œuvre ${ }^{2}$. » Dans Parerga et paralipomena il répète sa

\footnotetext{
* Martin Henry est professeur de théologie dogmatique à St Patrick’s College, Maynooth, Co. Kildare, Irlande.

${ }^{1}$ Une version anglaise de cet article a déjà paru dans la revue Irish Theological Quarterly, 65, 1, 2000, p. 3-12. Le présent article est une version révisée et légèrement augmentée de la version anglaise. Il a été traduit en français par Toby GARFITT (Magdalen College, Oxford), auquel l'auteur exprime ses vifs remerciements.

${ }^{2}$ Arthur Schopenhauer, Le Monde comme volonté et représentation, t. 1, livre 4, § 70, in Sämtliche Werke, Wolfgang F. vON LÖHNEYSEN, éd., Darmstadt, Wissenschaftliche Buchgesellschaft, 1974, t. 1, p. 550 [première édition 1819 ; le passage cité fut ajouté lors de la deuxième édition de 1844 ; voir ibid., p. 721]. Trad. A. BuRDEAU, nouvelle édition revue et corrigée par R. Roos, Paris, Presses Universitaires de France, 1966. George SANTAYANA, également, trouve évidente l'identification du péché originel avec la volonté : «L'existence en soi n'est pas un bien, mais seulement une occasion. Les chrétiens rendent grâces à Dieu de leur création, préservation, et toutes les bénédictions de cette vie, mais la vie est la condition et la source de tout mal, et les Indiens rendent grâces à Brahma ou au Bouddha de les en avoir retirés. Ce qu’un psychologue métaphysicien appelle la Volonté est le grand péché originel, l'intérêt inexplicable et irrationnel que l'esprit (une fois qu'il est incarné) prend à ce qu'une chose arrive plutôt qu'une autre [...]", George SANTAYANA, "Carnival », in Soliloquies in England and Later Soliloquies, Londres, Constable, 1922, p. 139 sqq. L'existence d'un lien
} 
définition du christianisme : «L’augustinisme avec sa doctrine du péché originel et tout ce qui s’y rapporte, [...] voilà le véritable christianisme, pour peu qu’on le comprenne bien ${ }^{3}$. »

Plus près de nous, le pessimiste Cioran, comme Schopenhauer, désigne le péché originel comme l'élément essentiel du christianisme.

L’éminent ecclésiastique se gaussait du péché originel. «Ce péché est votre gagne-pain. Sans lui, vous mourriez de faim, car votre ministère n'aurait plus aucun sens. Si l'homme n’est pas déchu dès l’origine, pourquoi le Christ est-il venu ? pour racheter qui et quoi ? » À mes objections, il n’eut, pour toute réponse, qu'un sourire condescendant.

Une religion est finie quand seuls ses adversaires s'efforcent d'en préserver l'intégrité ${ }^{4}$.

Entre ces deux penseurs - du point de vue chronologique mais non philosophique - il y a Nietzsche, d'abord grand admirateur de Schopenhauer, mais qui finit par en devenir un critique sévère. Pour Nietzsche, comme pour Schopenhauer, le christianisme appuyait et même encourageait une vue sombre de la vie terrestre, mais à la différence de son aîné, il le flétrissait pour l'antagonisme qu'il lui trouvait envers la vie ${ }^{5}$ et la culture ${ }^{6}$. Il est vrai que Nietzsche se sert rarement du terme « péché originel $^{7}$ » sauf de manière oblique ou équivoque

inaliénable entre le péché originel et le libre-arbitre fait bien partie de la doctrine catholique, même si ce lien n'est pas conçu de la même manière que chez Schopenhauer ou Santayana.

${ }^{3}$ Arthur Schopenhauer, Parerga und Paralipomena, t. 2, [1851], § 180, in Sämtliche Werke, op.cit., t. 5, 1976, p. 457.

${ }^{4}$ Emile Michel CiORAN, Aveux et anathèmes, Paris, Gallimard, 1987, p. 29.

5 «Ce n'est pas cela qui nous distingue, le fait que nous ne retrouvions aucun Dieu, ni dans l'histoire, ni dans la nature, ni derrière la nature - mais c'est que loin de ressentir comme divin ce qui fut vénéré comme Dieu, nous le ressentons au contraire comme pitoyable, comme absurde, comme pernicieux, non plus seulement comme une erreur mais comme un crime contre la vie [...] », Friedrich NiETZSCHE, L’Antéchrist, § 47, trad. Dominique TASSEL, Paris, Union Générale d’Éditions, 1997.

${ }^{6}$ « Le christianisme nous a privés de la moisson de la culture antique, plus tard il nous a encore privés de la moisson de la culture islamique », ibid., § 60.

${ }^{7}$ On trouve, par exemple, dans Humain, trop humain une référence en passant : le péché originel y est écarté sommairement (avec d'autres doctrines chrétiennes) comme illusoire, ce qui ne l'empêche pas d'être une puissante force historique. À propos de la Conférence de Ratisbonne, convoquée en 1541 afin de tenter une conciliation entre catholiques et protestants, Nietzsche écrit: "Il ne faut pas oublier qu'aucun des axiomes sur quoi l'on discutait alors à Ratisbonne ne possédait ombre de réalité, ni celui du péché originel, ni celui du salut par les intercesseurs, ni celui de la justification par la foi et qu'ils ne peuvent plus se discuter aujourd'hui. - Et pourtant, à cause de ces articles de foi, le monde fut mis à feu et à 
et afin d'ébranler le christianisme ${ }^{8}$. C'est ainsi que dans son « exégèse » du chapitre 3 de la Genèse, il emploie le terme avec beaucoup de sarcasme pour décharger son dégoût de ce qu’il considère comme l’hostilité du christianisme à l’égard de la science (« La Science est le premier péché, le germe de tous les péchés, le péché originel $\left.^{9} »\right)$; ou bien il en exploite le sens en exprimant son mépris de la théologie ${ }^{10}$; ou enfin il invoque la notion du péché originel lorsqu'il critique l'esprit prétendument rabat-joie du christianisme ${ }^{11}$. On ne trouvera donc chez Nietzsche aucune discussion franche, sérieuse ou soutenue du dogme théologique du péché originel ; mais plutôt le rejet énergique de toute appréciation pessimiste de l'existence humaine, appréciation qu'on a l'habitude de représenter depuis longtemps, dans la culture occidentale, par la formule consacrée de « péché originel ».

On pourrait se demander ici si la critique violente que Nietzsche adresse au christianisme pour l'aversion qu'il ressentirait pour la vie ${ }^{12}$ et la manière dont elle se transmet ne s'appliquerait pas plutôt au seul augustinisme (pour peu qu'on puisse le distinguer du christianisme proprement dit), qui soulignait l’importance du péché originel pour l'existence humaine. Il est vrai que Nietzsche avait en horreur la notion inquiétante que le péché originel se transmettait par l'acte de procréation. Mais cette notion, qui apparaît avec Tertullien et atteint son expression définitive (selon l'opinion générale) chez saint Augustin, ${ }^{13}$ manque de

sang. On se battit donc pour des opinions qui ne correspondent à rien de concret ni de réel [...] ", Friedrich NiETZSCHE, Humain, trop humain, t. 2, première partie, "Opinions et maximes variées ", § 226, trad. A.-M. DesRousseaux et H. Albert, revue par Angèle KREMER-MARIETTI, Paris, Livre de Poche, 1995).

${ }^{8}$ Cf. L'Antéchrist, § 61 : « La vieille corruption, le peccatum originale, le christianisme. » ${ }^{9}$ Ibid., § 48.

10 « La théologie, c’est la perversion de la raison par le "péché originel” (le christianisme) », Friedrich NiETzSche, Le Crépuscule des idoles, "Flâneries inactuelles », § 2, trad. Henri AlBERT, Paris, Garnier-Flammarion, 1985.

11 «Depuis qu'il est des hommes, trop peu s'est réjoui l'homme : c'est là seulement, mes frères, notre péché originel ! », Friedrich NiETzsche, Ainsi parlait Zarathoustra, deuxième partie : « Des compatissants », trad. Maurice DE GANDILLAC, Paris, Gallimard, 1971.

12 Selon Nietzsche, les premiers chrétiens qui finirent par prendre le contrôle de l'empire romain et le « sucer », étaient « des vampires rusés, sournois, invisibles, assoiffés de sang ! », in L'Antéchrist, op. cit., § 59.

13 « Tertullien, saint Cyprien et saint Ambroise enseignaient la solidarité de la race humaine entière avec Adam, non seulement dans les conséquences de son péché mais dans le péché lui-même, qui est transmis par la génération naturelle [...] », The Oxford Dictionary of the Christian Church, sous la direction de F. L. Cross et E. A. Livingstone, $3^{\mathrm{e}}$ éd., Oxford, OUP, 1997, p. 1196. La contribution de saint Augustin (un "agitateur chrétien », d'après Nietzsche, L'Antéchrist, § 59) au développement de la doctrine du péché originel et à l'intime liaison de celle-ci avec le sexe a souvent été mise en lumière : voir, par exemple, la discussion 
preuves néo-testamentaires.

On peut donc soutenir - mais la question n'est pas tranchée ${ }^{14}$ - que c'est l'interprétation augustinienne du péché originel ${ }^{15}$ qui est en jeu lorsque Nietzsche formule sa critique si véhémente de l'attitude chrétienne à l'égard des relations sexuelles: "Ce n’est que le christianisme, avec son fond de ressentiment contre la vie, qui a fait de la sexualité quelque chose d'impur : il jette de la boue sur le commencement, sur la condition première de notre vie $[\ldots]^{16}$ ». Dans ce contexte il est intéressant de voir Nietzsche verser au dossier le dogme

très utile de ce point in Peter Brown, Augustine of Hippo, Londres, Faber \& Faber, 1967, p. 387 sqq. Il convient pourtant de noter qu'Augustin semble avoir été plus démocratique que Tertullien, pour qui, si « toute âme est touchée par le mal par une faute d'origine, ce mal est attribué non à son appartenance à la race d'Adam, mais à une contamination païenne avant et après la naissance », G. WATSON, recension d'Augustinus-Lexikon, t. 1, fasc. 4, sous la direction de C. MAYER et alii, Bâle/Stuttgart, Schwabe \& Co/AG, 1990, parue in ITQ, 58, 1, 1992, p. 82.

${ }^{14}$ Ce serait trop nous écarter du sujet que de se demander si Nietzsche est tout à fait digne de foi quand il s'agit de l'histoire des attitudes chrétiennes envers la sexualité. Ses remarques polémiques et trop générales sont sans doute à prendre comme révélatrices du dégoût que lui inspiraient les mœurs de sa propre société plutôt que comme des indications très précises d'attitudes historiques. Quand il s'en prend à des figures du passé, son intérêt principal est d'ailleurs loin d'être historique ; c'est plutôt que de telles figures symbolisent opportunément des attitudes qu'il a en horreur dans le présent et qu'il considère comme étant typiques de la culture chrétienne. Quant à saint Augustin, ce serait déformer sa pensée que de supposer que d'instinct il haïssait le corps ou l'ordre matériel en soi, ou qu'il s'en méfiait (voir l'analyse méticuleuse de Peter BrOWN). Ce qui dominait la pensée d'Augustin était la conviction inébranlable que la chute représentait un acte de désobéissance de la part d'Adam, où l'âme agissait en désaccord avec le corps, dont la volonté n’arrivait pas à diriger les actions. Ce désaccord se reflétait dans la façon dont la volonté était désormais incapable de maîtriser les pulsions sexuelles, tandis qu'au paradis elle aurait pu le faire (théoriquement) avant la chute. Mais pour Augustin la «concupiscentia carnis » n'est nullement limitée au domaine sexuel : "La concupiscence était un désir maléfique de maîtriser, d'accaparer, et de détourner à ses propres fins toutes les bonnes choses que Dieu avait créées pour être reçues avec reconnaissance et partagées avec les autres. Elle était à la racine de la misère dont les hommes étaient partout et inévitablement accablés. Le désir spécifiquement sexuel n’était pas plus entaché de cette concupiscence tragique et sans visage que n'importe quelle autre forme d'activité humaine.", Peter Brown, The Body and Society: Men, Women and Sexual Renunciation in Early Christianity, Londres, Faber \& Faber, 1990, p. 418.

${ }^{15}$ Sur les complexités indéniables de la position augustinienne à l'égard de la transmission du péché originel, voir John M. RIsT, Augustine: Ancient Thought Baptized, Cambridge, CUP, 1994, appendice 2 : «Traducianisme, créationnisme et péché originel », p. 317-320. Malgré les obscurités persistantes, Rist conclut ainsi : «Le lien entre les rapports sexuels (déchus) et la transmission du péché originel ne fait aucun doute »; c'est seulement la manière dont « la “qualité immatérielle” du péché » pouvait être transmise par " les rapports sexuels déchus » qui restait mystérieuse pour Augustin, ibid., p. 319.

${ }^{16}$ Friedrich Nietzsche, Le Crépuscule des idoles, "Ce que je dois aux anciens », § 4 (cf. Walter KaUfmanN, Nietzsche: Philosopher, Psychologist, Antichrist, Princeton, Princeton University Press, $4^{\mathrm{e}}$ éd., 1974, p. 223). 
de l'Immaculée Conception, mais mal le comprendre comme cela a souvent été le cas ${ }^{17}$. Lorsqu'il demande: «Est-il permis d'être chrétien aussi longtemps que la notion d'immaculata conceptio sert à christianiser, c'est-à-dire à souiller l'origine de l'homme ${ }^{18}$ ? ", il semble - comme dans un passage antérieur du même ouvrage où il prétend qu'avec « un dogme de "conception immaculée” », le christianisme « a par le fait maculé la conception ${ }^{19}$ " - avoir confondu la doctrine de la conception virginale de Jésus avec celle de l'immaculée conception de Marie $^{20}$.

L'erreur de Nietzsche est ironique, car le dogme de l'immaculée conception va dans le sens même où il voulait à tout prix aboutir. Ce n'est pas tomber dans l'ergotage que de relever son erreur. En définissant la conception de Marie comme « immaculée », et en affirmant ainsi qu'elle était exempte du péché originel, la doctrine catholique niait l'existence d'un lien intrinsèque entre celui-ci et l'acte de procréation. En deux mots, dans le cas de Marie il y eut acte de procréation, mais non transmission de péché originel. Ainsi, quelle que soit la source ou la nature du péché originel dont Marie fut préservée, on ne peut pas la localiser dans l’acte de génération lui-même. L’objet de la doctrine de l’Immaculée Conception est précisément d'affirmer que Marie est toujours, dès l'origine (dès la « conception ») de sa vie, exempte du péché originel ; tout comme l’objet du dogme du péché originel est de souligner que toute la race humaine est concernée, dès l'origine de la communauté humaine. Il était sans doute inévitable qu'on en vienne à mettre l'accent sur le mot " conception »), avec sa forte charge sexuelle, tout comme on l'a mis, dans le cas du péché originel, sur la notion des origines de la vie humaine, notion également liée (de manière universelle, du moins jusqu’à ces derniers temps) à la pulsion sexuelle.

Bien sûr, il serait trompeur de nier le lien qui a souvent été fait, ou supposé, entre le péché originel et l'origine sexuelle de l'être humain. La croyance à ce lien - confuse et porteuse de confusion - a sans aucun doute embrouillé les attitudes chrétiennes à l'égard de la sexualité tout au long des siècles ${ }^{21}$. L'histoire d'Adam et d'Ève, de Dieu et du Serpent dans le jardin

\footnotetext{
17 Voir, par exemple, Luis Buñuel, My Last Breath (Mon dernier soupir), trad. Abigail ISRAEL, Londres, Vintage, 1994, p. 44.

${ }^{18}$ Friedrich NiETZSCHE, L'Antéchrist, op. cit., § 56.

${ }^{19}$ Ibid., § 34.

${ }^{20}$ Cf. le commentaire sur L'Antéchrist, § 34, in Friedrich NIETZSCHE, Sämtliche Werke: Kritische Studienausgabe, t.14, G. Colli et M. MonTINARI, dir., Munich/Berlin, DTV/de Gruyter, 1988, p. 442.

${ }^{21}$ Soutenir cela n'est pas prétendre, loin de là, que si le lien entre le péché originel et la sexualité humaine était brisé, celle-ci retrouverait toute son innocence. Le péché originel, en tant que doctrine qui concerne la condition historique de l'homme par rapport à Dieu, n'en
} 
d'Éden, a préoccupé toutes sortes d'esprits au cours des siècles, mais comme le dit un commentateur recent : «La sexualité [...] n’a pas cessé de se glisser indûment dans les interstices de l'histoire. Selon Augustin, le fruit de l'arbre était un aphrodisiaque, qui entraîna la conquête de notre volonté par la luxure. Beaucoup d'artistes, dont Dürer, ont mis un chat prêt à bondir sous l'arbre dont nos parents ont mangé ; le chat (respecté par les musulmans) symbolisait le désir sexuel dans la littérature chrétienne primitive ${ }^{22}$ ».

Le dogme de l'Immaculée Conception aurait dû servir de barrière contre toute identification erronée et inutile de la transmission du péché originel avec la procréation, mais il semble avoir plutôt conforté cette identification. La confusion avec la conception virginale de Jésus, mentionnée plus haut, confusion qui se produit si souvent et pour ainsi dire naturellement, en serait une preuve. Elle tend à renforcer dans la croyance populaire le pressentiment que la sexualité en soi est impure, et qu'étant indéracinable elle doit être la cause des maux perpétuels de l'humanité. Bref, la felix culpa, l’heureuse faute, a eu une histoire des plus malheureuses.

Plutôt que la sexualité, c’est sans doute le rôle de la liberté humaine dans l'économie du salut qui serait l'objet du dogme du péché originel ${ }^{23}$. En effet, ce dogme affirme non seulement que les conséquences des décisions humaines libres peuvent être catastrophiques, mais qu'elles peuvent également, d’une manière mystérieuse, mener à un bien supérieur à celui qui avait été perdu à cause du péché. Ou, pour parler sur le plan du mythe, la merveille du paradis s’étant avérée illusoire - Nietzsche dit malicieusement « ennuyeuse ${ }^{24}$ » l’humanité est ainsi amenée à chercher ailleurs la plénitude. Que l’idée même du paradis puisse être ennuyeuse est implicite dans ces vers bien connus de Goethe : «Tout au monde peut être supporté, à l'exception d'une série de belles journées ${ }^{25}$. " Elle avait déjà été exprimée par le Réformateur Martin Luther ${ }^{26}$, ce qui n’est sans doute pas étonnant, et elle fut reprise par la suite par Hegel qui, comme Nietzsche, était lui-même profondément pénétré de

continuerait pas moins de toucher tous les aspects de l'existence humaine, y compris la sexualité ; mais le lien indéfendable entre les rapports sexuels et la transmission du péché originel disparaîtrait.

22 Robin Lane Fox, The Unauthorized Version: Truth and Fiction in the Bible, Harmondsworth, Penguin, 1992, p. 26.

${ }^{23}$ Voir la note 2 ci-dessus.

${ }^{24}$ Friedrich NIETZSCHE L'Antéchrist, op. cit., § 48.

25 «Alles in der Welt läßt sich ertragen,/Nur nicht eine Reihe von schönen Tagen », Johann Wolfgang VON GOETHE, « Keins von allen », in Sprüche.

26 Voir Georg BüCHMANN, Geflügelte Worte, 39 édition revue par W. Hofmann, Francfort/Berlin, Ullstein, 1993, p. 128. 
la tradition luthérienne ${ }^{27}$. L'expulsion du paradis s'avère pourtant non un désastre complet, mais plutôt le passage obligatoire qui permettra à l’humanité d'atteindre Dieu.

Ce langage n'est pas celui de l'histoire ou de la science, bien entendu, mais celui de la foi et de l'espérance. C’est le péché originel qui rend certaines de nos réactions à l'univers que nous nous trouvons habiter insuffisantes au point d'être nuisibles (comment pourrait-il en être autrement, puisque ce n’est pas nous qui avons créé le monde ?). Mais en même temps aucune faute n’est si irrémédiable que Dieu ne puisse pas s’en servir pour notre bien, à la longue. Dire cela ne fait pas des êtres humains de simples jouets du destin ou de Dieu. Au contraire, la croyance au péché originel souligne notre liberté ainsi que notre importance, en ce qu'elle fait reposer la responsabilité de notre situation sur nous-mêmes, ${ }^{28}$ tout en laissant entendre qu'il y a une réalité au-delà de notre responsabilité, car s’il n’en était pas ainsi, devant qui ou quoi nous sentirions-nous responsables ? Cette prise de conscience relativise notre situation, sans nous abaisser, et nous fait goûter le soulagement d’être tolérés, peut-être même désirés et voulus, par ce qui nous transcende totalement. En d'autres termes, le péché originel n’est pas un symbole de l'horreur de l'existence, comme si nous n'étions pas à notre place ici. Adélard de Bath était sans doute plus près de la vérité que beaucoup de pessimistes exubérants à travers les âges quand il écrivit au XXII ${ }^{\mathrm{e}}$ siècle que « si les humains négligent de connaître la beauté de l'univers où ils vivent, ils méritent d'en être expulsés comme des hôtes incapables d’apprécier une maison où l'hospitalité leur est offerte ${ }^{29}$ ».

La façon dont Adélard représente la condition humaine est manifestement assez différente de celle de la plupart des autres penseurs chrétiens, qu'il soient antérieurs ou postérieurs. Il est possible que dans l'Église primitive certains aient été amenés par leur désir de réfuter les gnostiques - qui identifiaient la création avec la chute - à trop accorder à leurs adversaires,

\footnotetext{
${ }^{27}$ Hegel écrit non que le paradis soit précisément ennuyeux, mais que « l'état d’innocence, la condition paradisiaque, appartient à la brute. Le paradis est un parc naturel, où les brutes seules, et non les hommes, peuvent rester ", in Friedrich Hegel, Philosophie de l'histoire, cité par James C. Livingston, Modern Christian Thought. From the Enlightenment to Vatican II, New York, Macmillan, 1971, p. 151).

${ }^{28}$ Cf. Gregory Shaw, art. "The Fall », in Bruce M. MetZger et Michael D. Coogan, The Oxford Companion to the Bible, Oxford, OUP, 1993, p. 223 : « Le mythe biblique de la chute est semblable à d'autres légendes qui contrastent l'actuel état misérable de l'humanité avec une ère antérieure de perfection, paradis perdu ou âge d'or. Le récit biblique est toutefois unique lorsqu'il implique que la dégradation de l'humanité était le résultat indirect d'un choix libre. »

${ }^{29}$ Cité par Matthew Fox, « Afterword to the 1990 Edition », in Original Blessing, Santa Fe, Bear \& Company, $1^{\text {re }}$ édition 1983, d'après Marie-Dominique CHENU, Nature, Man and Society in the Twelfth Century, Chicago, University of Chicago Press, 1968, p. 13.
} 
dans l'espoir de convaincre les indécis qui auraient été attirés par l’analyse gnostique de certaines vérités inconfortables mais indéniables de l'existence. On ne peut pas cacher d'importantes ressemblances entre les premiers chrétiens et leurs rivaux gnostiques, et ces ressemblances ne doivent pas nous surprendre, car les deux camps se disputaient le même territoire et se voyaient confrontés aux souffrances du même monde. Le christianisme, comme le gnosticisme (mais avec plus de réserves, du moins sur le plan doctrinal), se teinta du pessimisme de la fin de l'Antiquité ${ }^{30}$. Il importe finalement peu que, du haut de notre $\mathrm{XXI}^{\mathrm{e}}$ siècle, nous mettions la tendance pessimiste de la culture chrétienne sur le compte du vieux combat contre le gnosticisme ou sur celui du judaïsme ou de l'hellénisme, car la condition humaine a toujours offert des motifs de «broyer du noir » à ceux qui aiment le pessimisme et le désespoir.

Ce serait quand même, à mon avis, trahir la pensée d'Augustin que de suggérer - comme on est parfois tenté de le faire - que c'est lui qui a injecté dans le courant sanguin de l'Occident un pessimisme toxique qui depuis lors convulse notre culture de temps en temps ; ce serait méconnaître, d'une manière presque injurieuse, la subtilité pénétrante de cet esprit jamais en repos, la grande vision conductrice de sa foi et de son espérance. Même dans ses dernières années, années «de frustration amère », nous dit Peter Brown, « Augustin n’abandonna jamais l'espoir d'après lequel il jugeait - de plus en plus tristement - la misère présente de la race humaine. C’était toujours un espoir d’unité. L’harmonie brisée serait rétablie à la fin des temps. Dans ses sermons nous percevons l'élan d'un cœur inlassable. Le défaut fatal de la concupiscence n’aurait pas semblé si tragique à Augustin s’il ne s'était pas toujours plus fermement persuadé que les êtres humains avaient été créés pour embrasser le monde matériel. Si le corps était un problème pour lui, c'est précisément parce que le corps était fait pour être aimé et chéri ${ }^{31}$. » Un témoignage important de son affirmation de la vie est fourni par son «constant aplomb littéraire ${ }^{32}$ », que cet homme qui prenait tant de plaisir évident à écrire ne devait jamais perdre, même in extremis. Il est donc un peu injuste envers le Docteur de la Grâce (mais en même temps tout à fait compréhensible) de conclure, avec Dodds, que ce ne fut malheureusement « pas Origène mais Augustin qui traça la voie future à

${ }^{30}$ Cf. Basil Willey, Christianity Past and Present, Cambridge, CUP, 1952, p. 69 : « Il y a peu de doute que les doctrines trouvent souvent leur coloration, et leur puissance, dans les circonstances historiques. On a pu dire, par exemple, que la doctrine du péché originel dérive de la Weltschmerz antique. L'œuvre de saint Augustin fut contemporaine du sac de Rome en 410, et au moment de sa mort les Vandales étaient aux portes d'Hippone. L'expression la plus importante de cette doctrine était donc due à une époque de confusion et de désespoir. »

${ }^{31}$ Peter Brown, The Body and Society, op. cit., p. 425.

${ }^{32}$ Ibid., p. 423. 
l’Occident chrétien ${ }^{33} »$.

Ce fut donc sous l'impulsion sans doute inégale de l'irrésistible génie d'Augustin (car qui a le temps ou la force d'être entièrement impartial ?) que le christianisme occidental absorba volontairement, voire avec enthousiasme, le pessimisme de la culture de la fin du monde antique, et le rattacha par le baptême, à l'usage des générations à venir, au concept du péché originel. Il est ironique que ce concept précisément ait pris tant d'importance dans la culture chrétienne, puisque «l’idée que quelque grand péché explique la misère de la condition humaine était partagée par les païens et les chrétiens dans l’Antiquité tardive ${ }^{34}$ », tandis que le christianisme primitif s'était justement proposé de se présenter comme un nouveau commencement et de se différencier clairement du monde païen. Mais « faute de grives on mange des merles » : il est toujours nécessaire de trouver un solide point de contact entre le message chrétien et la culture à laquelle il s’adresse, et résister à la tentation de faire appel au péché originel pour recommander le christianisme au monde d'alors aurait sans doute été inutilement scrupuleux.

À l'ombre de l'imposante, de l'incontournable œuvre d'Augustin, le christianisme occidental allait vivre l'existence pratiquement comme une expiation du crime de naître, dont la mort promettait une délivrance vers le ciel pour quelques élus, et la menace d'une descente en enfer pour la «massa damnata ${ }^{35} »$. Ce monde-ci n’était qu’une vallée de larmes qu’il

${ }^{33}$ E.?? R. DodDs, Pagan and Christian in an Age of Anxiety, Cambridge, CUP, 1965, p. 132. Chose curieuse, comme Dodds lui-même l'avait déjà indiqué (p. 24, n. 4), tant le chrétien Origène, que le païen Plotin, ne célébraient jamais leur anniversaire, puisque tous les deux considéraient le fait de naître comme « un Malheur ». Qui étaient alors les vrais pessimistes ?

${ }^{34}$ Peter Brown, Augustine of Hippo, op. cit., p. 388. Brown mentionne Cicéron (voir Contra Julianum, IV, xiii, 78), et pour illustrer davantage ce point, il cite De moribus ecclesiae catholicae, I, xxii, 40 : «Le Péché ancien : rien ne fait plus évidemment partie de notre prédication, et pourtant rien n'est plus impénétrable à l'entendement. »

${ }^{35}$ Ceci fit l'objet d'une réaffirmation officielle au $\mathrm{XV}^{\mathrm{e}}$ siècle, comme nous le rappelle David L. EDWARDS : «En 1439 le concile de Florence enseigna que tous les adultes non baptisés doivent nécessairement aller en enfer même s'il n’ont commis aucun péché personnel, parce qu'ils ont hérité le péché “originel” d'Adam » in Christianity: The First Two Thousand Years, Londres, Cassell, 1997, p. 175. L'enfer comportait toutefois des gradations, selon ce même concile, tout comme le ciel : «Les âmes de ceux qui quittent cette vie en état de péché mortel réel, ou en état de péché originel tout seul, descendent immédiatement en enfer pour être punis, mais avec des peines inégales. ", Norman P. TANNER, S.J., éd., Decrees of the Ecumenical Councils, Londres/Washington D. C., Sheed \& Ward/Georgetown University Press, 1990, t. 1, p. 528 ; cf. ibid., p. 578, où nous lisons que le concile «croit fermement, professe, et prêche que tous ceux qui sont en dehors de l'église catholique, non seulement les païens mais aussi les juifs, les hérétiques et les schismatiques, sont privés de la participation à la vie éternelle, et iront dans le feu éternel préparé pour le diable et ses anges [Mt 25, 41], à moins d'être joints à l'église catholique avant la fin de leur vie [...] ». La version calviniste 
fallait traverser avant d'arriver à quelque chose de meilleur, comme ne cessaient de l'asséner d'innombrables exhortations sur le thème du contemptus mundi. Le fameux pessimisme des Réformateurs au $\mathrm{XVI}^{\mathrm{e}}$ siècle ainsi que des jansénistes et des puritains au XVII ${ }^{\mathrm{e}}$, qu'on évoque à toute occasion, représente bel et bien une des filières de l'augustinisme, et sa sévérité offre un contraste frappant avec certaines des aspirations plus joyeuses du XVIII ${ }^{\mathrm{e}}$ siècle.

Mais les moments joyeux de la culture occidentale semblent bien être l'exception plutôt que la règle. Il n’est guère étonnant que le philosophe sceptique George Santayana, fortement imbu de culture catholique, se soit demandé si même les grands mystiques chrétiens n’étaient pas au fond comme des nihilistes cachés, opposant un refus à la vie : « La croyance au ciel n’a-t-il pas souvent été un désir de ne pas vivre, plutôt que de vivre éternellement ? Je suis tenté de le croire. Vous connaissez le vers de sainte Thérèse et de saint Jean de la Croix : "Muero porque no muero" ${ }^{36}$."

Et pourtant une fausse interprétation d'une croyance - même voulue - ne l'infirme pas forcément tout entière. Rejeter l'interprétation du péché originel qui le lierait trop exclusivement à la dimension sexuelle de la nature humaine, ou qui le verrait comme autorisant un pessimisme intégral, n'est pas vider cette doctrine de toute signification. Au contraire, cela invite à trouver une meilleure interprétation que celle qui a été ainsi écartée. Par exemple, ce serait proposer non de remplacer le péché originel par «la bénédiction originelle $^{37}$ " mais de le mieux comprendre, à la manière de Kolakowski, comme une invitation à «nous faire prendre conscience de la contingence et de la finitude de la vie humaine, de la corruptibilité du corps, des limitations de la raison et du langage, du pouvoir du mal en nous ${ }^{38}$ ». Dans une telle perspective, le péché originel indique à la fois le besoin du rachat et sa possibilité. Le besoin saute aux yeux ; quant à la possibilité, qu’elle soit réalisée ou non ne dépend pas uniquement de nous, car nous ne sommes pas seuls en jeu. Mais cette possibilité du rachat, que le dogme du péché originel s'efforce d'indiquer - d'une manière gauche et ambiguë, certes, mais peut-être non sans pertinence de ce fait même -, a pu être entrevue pour la première fois à travers le jugement flou porté par le christianisme sur l'existence humaine, jugement exprimé dans la personne de Jésus, l’innocente victime qui

de la Bonne Nouvelle comme double prédestination se faisait encore attendre : c'est elle que certains considèrent comme la dernière expression, intransigeante et sans équivoque, de la théologie augustinienne de la grâce (voir Jaroslav PELIKAn, The Melody of Theology, Cambridge, Mass., Harvard University Press, 1988, p. 108 sqq.)

${ }^{36}$ The Letters of George Santayana, édition de Daniel Cory, Londres, Constable, 1955, p. 395 (lettre à Corliss Lamont, 8 juin 1950).

${ }^{37}$ Croyance qui s’est traditionnellement exprimée par la doctrine de création.

${ }^{38}$ Leszek KolAKOWSKI, Religion, Oxford, OUP, 1982, p. 200. 
rachète notre humanité.

Pour le christianisme, l'homme est pris entre l'impossibilité d'atteindre le bonheur par luimême, et celle (également réelle) de renoncer définitivement à la quête du bonheur. Le péché originel, qui empêche d'atteindre ce bonheur d'une manière délibérée, et la nature indéterminée de l’homme (« fait à l'image de Dieu »), qui laisse ouverte la possibilité d'une recherche acharnée mais jamais finie du même bonheur, sont deux constantes de l'existence qui expliquent l'éternel retour de la tragédie dans l'histoire : «Es irrt der Mensch, so lang er strebt », comme le dit Goethe ${ }^{39}$.

S’il est important de voir le péché originel en fonction du rachat, il ne l'est pas moins de le voir en fonction de la création. Cela lui rend sa véritable signification qui est de mettre en lumière le rapport primordial et indissoluble de l'homme avec Dieu, quels que soient les événements de l'histoire. Pour ceux qui ont des yeux pour voir, ce rapport sera vécu le plus souvent - puisque nous croyons que Dieu est bon - comme un «manque à atteindre » la gloire de Dieu ${ }^{40}$. Là est la vraie chute, non dans quelque catastrophe pré-cosmique ou préhistorique que nous avons été incapables d'empêcher et pour laquelle nous devons être punis éternellement. Mais « manquer d'atteindre » n’empêche pas d’être vaguement conscient de la bonté qui non seulement nous attend, mais qui est à la base de tout et embrasse tout. Cette bonté ne nous appartient manifestement pas, même si nous la sentons, et c'est pourquoi dans la tradition chrétienne on l'appelle généralement la grâce. C’est parce que la grâce ne cesse pas d'être, du moment que nous ne la possédons pas, qu'il est raisonnable de croire au rachat. Le soulagement de savoir qu'il y a quelque chose qu'on «manque d'atteindre », voilà peutêtre le sens de la paix religieuse. Le soupçon que ce « manque à atteindre » sera éternel, que nous sommes destinés à partager le sort de Tantale, peut obnubiler cette paix mais n’a pas le pouvoir de la détruire. À chaque univers suffit sa clarté, fût-ce un « nuage d'inconnaissance ».

Cet univers où nos vies se déroulent révèle sa vraie valeur, et non son manque de valeur, à la lumière du péché originel. La «tristesse majestueuse ${ }^{41}$ » de l'histoire humaine ne correspondrait à rien si la vie humaine n’avait aucune importance. Car, tout paradoxal que cela puisse paraître, l'importance de notre vie est soulignée et non niée par le fait que nos

39 Johann Wolfgang VON Goethe, Faust, $1^{\text {re }}$ partie : «Prologue au ciel », trad. Henri LiCHTENBERGER, Paris, Aubier, 1952. « L'homme erre tant qu'il s'efforce et cherche. »

${ }^{40}$ Cf. Rm 3, 23.

41 Jean Racine, Bérénice, Préface. Comme me l’a signalé le Professeur John Campbell, de l’Université de Glasgow, je détache ici l'expression de son contexte originel, Racine évoquant la « tristesse majestueuse qui fait tout le plaisir de la tragédie ». 
décisions peuvent occasionner la souffrance. Cette découverte effrayante, narrée de façon paradigmatique par l'auteur de la Genèse, est re-présentée par tous les grands maîtres du tragique, et ne peut évidemment pas être totalement expliquée dans la langue de tous les jours. Pour la foi chrétienne, l'utilité du dogme du péché originel est qu’il constitue une démonstration oblique de la valeur indestructible de cette création au sein de laquelle nous avons « la vie, le mouvement et l’être », et en même temps le « péché originel » est la clé qui ouvre la porte de sortie du paradis et nous permet ainsi d'emprunter la route difficile vers le ciel $^{42}$.

Martin HENRY St Patrick's College, Maynooth, Co. Kildare, Irlande

${ }^{42}$ Pour certaines des idées contenues dans cet article je reconnais volontiers ma dette envers John CAMPBELL, "Racine and the Augustinian inheritance : the case of Andromaque ", in French Studies 53, 3 juillet 1999, p. 279-291. Dans cet article il réfute vigoureusement la traditionnelle lecture janséniste de Racine. 